\section{Inner machinery of the silicon chip}

\section{Andrew Holmes-Siedle}

Instabilities in MOS Devices. By J.R. Davis. Pp.190. ISBN 0-677-05590-9. (Gordon \& Breach: 1982.) \$24.50.

MosT silicon integrated circuits are smaller in size than a fingernail and contain many thousands of working elements, the most common of which is the metal-oxide-semiconductor (MOS) transistor. In this, all of the important electronic activity takes place in a minute volume, a layered structure composed of crystalline silicon, thermally-grown silicon dioxide and a conductor. Attempts are always being made to reduce the unit size of the transistor element, but during such development it is crucial to maintain the performance of the intimately interwoven materials of the layered structure. Instabilities in MOS Devices is a discussion of the physical processes occurring in this metal-oxide-silicon system.

In MOS structures under electrical fields, electrons, holes and ions sometimes migrate. This movement produces the instabilities in electrical properties referred to in the title. In their constant endeavour to ensure that instability effects do not wreck new designs, physicists regard the silicon dioxide layer as the key element; it is a complex film but, in this work, can often be regarded as a simple, wide-band-gap insulator region, sandwiched between the "clectron sea" of a metallic electrode and the "bendable" energy bands of the semiconductor. In this context, the instabilities are interpreted either as the unwanted carrying of holes and electrons across the two high, interfacial potentialenergy barriers by tunnelling or carrier excitation; or the internal drift of various ionic species, often sodium or hydrogen. The transport medium can often be regarded as very pure fused silica, which allows us to use the large fund of knowledge of the physics of crystalline and fused quartz - makers of science policy might note that this is a good example of the benefits imparted to technology by apparently unrelated basic research.

The understanding of charge movement in silica may seem to be a somewhat esoteric field; but, for obvious reasons, it is increasingly important to convey this knowledge to electronics undergraduates and to the growing numbers of workers entering the broader field of the development of "electronic systems on a chip". This book fills such a need. It is, for example, the first time I have seen a large

- Another recent textbook on MOS devices is Oliver McCarthy's MOS Device and Circuit Design. The book, primarily aimed at students of electronic engineering, but also intended for practising engineers, is published by Wiley at $£ 19.50, \$ 44.95$. and carefully selected set of references on instability research.

Such research is carried out using a limited number of specialized electrical and optical techniques. These are outlined in 30 concise pages. The theoretical principles required include those of defect solid state physics, surface physics, lattice dynamics, conduction physics and molecular quantum mechanics, and treatment of these concepts is woven into the subsequent chapters. Among the research findings discussed are recent developments in surface states, mobile ions, polarization, hole trapping, electron trapping and electrical breakdown.

For many of the intended audience the book will be tough going. Readers are provided with reference to a few of the best, general solid-state technology textbooks, but the style of writing is somewhat flat and the graphics are unimaginative, coming, in the main, directly from research papers or other textbooks. Nonetheless, the author does address all of the important conduction and trapping effects which determine whether or not a given MOS device will be "good".

The exploitation of conduction and trapping effects in the MOS system is only just beginning, and the practical aspects of instability research reinforce the usefulness of a review such as that provided by $\mathrm{Dr}$ Davis. The use of new and more subtle effects in MOS devices not only ensures "good" (i.e. stable) devices, but also leads to ones which are "better" than those of rival makers. In this context, it must not be forgotten that microelectronic devices are used not only in parlour games but also in machine tools, racing cars and missiles. The country which possesses the fastest and best microcircuits will perhaps win not only commercial success but also more explicitly gladiatorial contests.

Andrew Holmes-Siedle is Consultant to the Physics Department of the Fulmer Research Institute, Stoke Poges, Buckinghamshire.

\title{
Statistical expertise on the shelf
}

\section{P.T. Saunders}

Biometry: The Principles and Practice of Statistics in Biological Research, 2nd Edn. By Robert R. Sokal and James F. Rohlf. Pp.859 plus tables. ISBN 0-7167-1254-7. (W.H. Freeman: 1981.) £19.50, \$31.95. Tables hbk £16.30, \$22; pbk £6.95, \$9.95.

For some years now, Sokal and Rohlf's Biometry has enjoyed considerable success as a statistics text for biologists. Knowing this, my first impressions of the second edition were less favourable than I had expected. While the opening chapters are written in an attractive enough style and deal with the right sort of material, I found them too long and diffuse. The authors seem so intent on ensuring that every aspect of the subject is covered that they leave the reader in danger of not seeing the wood for the trees. To give just one example, the crucial idea of the testing of hypotheses seems to get lost in a long section devoted mainly to Type I and Type II errors and power functions. It is not that the latter are not worth discussing, though they are relatively less important in a text in which derivations are omitted, but that the balance is wrong.

But while I have some reservations about the introductory material, the remainder of the book, which consists of detailed accounts of a large number of statistical techniques applicable to a wide variety of problems, is very good indeed. The authors make excellent use of boxes, set aside from the main text, in which they show precisely how each test is to be carried out. They provide sample calculations for the benefit of those readers who are uncomfortable with subscripts and summation signs; more mathematically minded users will find them invaluable for testing computer programs. There are also informal discussions of the ideas behind the tests and other relevant points.

A number of improvements have been incorporated into the new edition and some additional material has been included, mainly concerned with correlation and regression. Inevitably the coverage is still not complete; for example, the authors have judged that their intended readership will be able to manage without such topics as sequential testing and Bayesian techniques. They have also chosen to publish the statistical tables separately instead of binding them with the book, which has its advantages so long as you can keep the two together.

Biometry would not be my first choice for someone with no previous knowledge of statistics, though it is certainly well worth considering. I would, however, strongly recommend it for anyone who already knows a bit about the subject and now finds himself in need of something beyond what he was taught. It is not quite as good as having a friendly statistician across the hall, but it can come surprisingly close.

Peter Saunders is a Lecturer in Mathematics at Queen Elizabeth College, University of London. 